\title{
LES PRODUCTIONS DE L'ATELIER DE LOYASSE
}

\author{
Martine GENIN, Jacques LASFARGUES et Anne SCHMITT
}

\begin{abstract}
Résumé. L'atelier de Loyasse a été découvert il y a une trentaine d'années sur la colline de Fourvière, au centre de l'actuel cimetière de Loyasse. On ignore le volume exact et l'importance de cet atelier matérialisé par une seule fosse, fouillée de surcrô̂t dans des conditions particulièrement difficiles. Le mobilier céramique recueilli atteste cependant qu'on a fabriqué à Lyon des imitations de sigillée, des vases à parois fines lisses et peignées ainsi que des gobelets d'Aco présentant un registre de frises caractéristique. L'étude de ces productions et les comparaisons que l'on peut établir avec le mobilier des contextes lyonnais les plus anciens suggèrent que la phase d'activité de l'atelier s'inscrit dans les années 30-20 avant J.-C.
\end{abstract}

\begin{abstract}
The workshop of Loyasse was discovered thirty years ago, on the hill of Fourvière, in the graveyard of Loyasse. We don't know the real importance of this workshop, only known through a single pit, excavated in hard conditions. The wares found there gave evidence that terra sigillata imitations, thin-walled pottery and Aco beakers with typical friezes were made in Lyon. The study of these wares and comparisons with those found in the more ancient contexts in Lyon suggest that the activity of the workshop of Loyasse took place during the 30-20 B.C. period.
\end{abstract}

\section{OIRCONSTANCES DES DÉCOUVERTES}

Après les traces ténues de production céramique repéées en 1964 au pied de la montée de la Butte et surtout les fouilles de la Muette de 1966, l'archéologie lyonnaise était fortement sensibilisée aux problèmes d'ateliers de potiers. C'est ce qui explique sans doute que la microdécouverte de Loyasse ne soit pas passée inaperçue : $2 \mathrm{~m}^{2}$ explorés, et situés dans un quartier de Lugdunum où l'on ne s'attendait guère à observer une activité de ce genre.

C'est en effet sur la partie sommitale de la colline de Fourvière que l'atelier de Loyasse a été localisé (fig. 4), au centre de ce que les Lyonnais nomment "nouveau cimetière de Loyasse ", compris entre la rue Le-Châtelier, la rue P.-Jaricot et la rue C.-Gerlier très précisément dans la masse 4. Ce secteur a été exploré dans sa partie nord à l'occasion des travaux des HLM de la Sarra, mais les zones sud, est et ouest sont restées très protégées par le périmètre du réservoir d'eau, par le cimetière ou par les fortifications du XIX ${ }^{c}$ s. Les seules observations semblent indiquer que les niveaux antiques ont été fortement arasés et que seules des fondations très profondes ou des structures en creux sont conservées.
R. Pelletier, archéologue bénévole très actif dans les années soixante, est à l'origine de la découverte. Ayant observé que des tessons étaient mis au jour dans la masse 4 du cimetière à chaque sépulture, il en a assuré une surveillance pratiquement constante et c'est ainsi qu'il nous a signalé, en mai 1967, qu'une importante quantité de fragments de gobelets d'Aco venaient d'apparaître dans le creusement d'une de ces fosses.

L'inhumation devant avoir lieu immédiatement, nous avons disposé d'une heure environ pour intervenir au fond de la fosse. La profondeur était comprise entre $2 \mathrm{~m}$ et $2,20 \mathrm{~m}$, et la surface accessible de l'ordre de $2 \mathrm{~m}^{2}$. L'installation des parois interdisait tout élargissement.

Les terrassiers s'étaient arrêtés au niveau supérieur de la couche témoin de l'atelier et l'avaient même partiellement entamée. Épaisse d'une dizaine de centimètres au maximum, elle était composée d'un conglomérat de petits fragments, tessons, boulettes d'argile, morceaux de tubulures, collés par de l'argile et du sable. L'ensemble était relativement tassé, sans qu'il soit possible de savoir s'il s'agissait d'un sol de travail ou de circulation, ce que tendrait à prouver la petite taille des tessons, ou d'un dépotoir très étalé, mais compacté. La couche reposait directement sur un niveau d'argile stérile, peut-être le ter- 


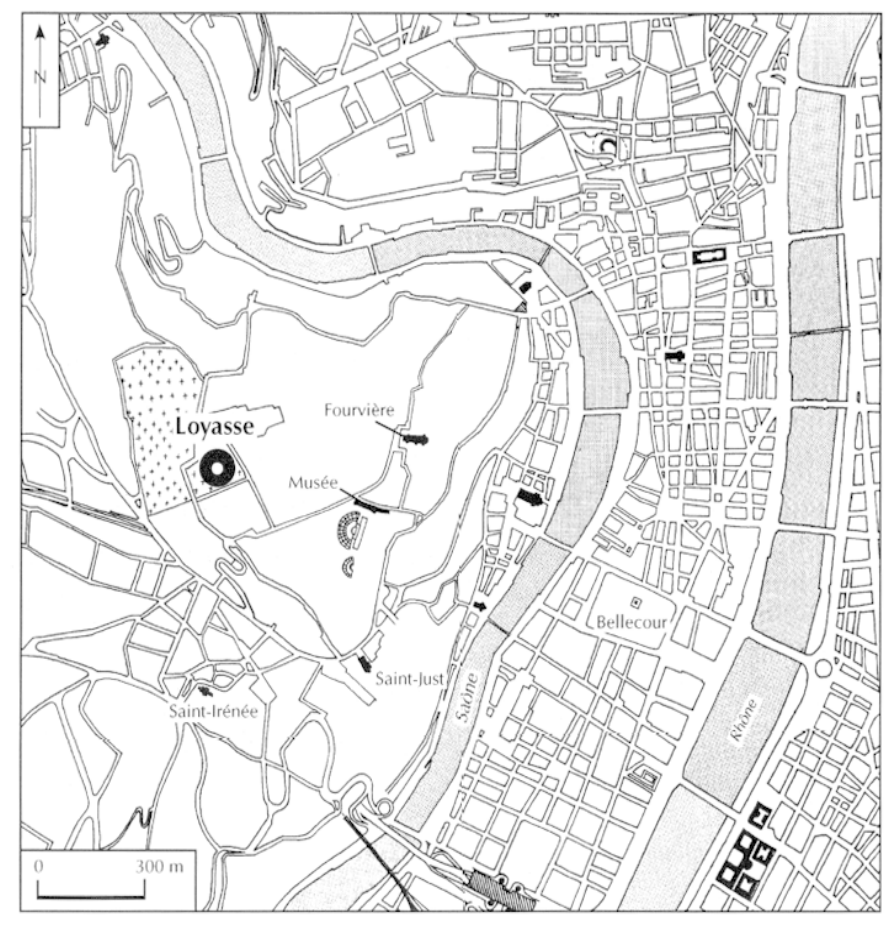

Fig. 4. Plan de localisation de l'atelier de Loyasse dans la ville antique.

rain naturel qui est souvent constitué, sur cette partie de la colline, par des bancs assez puissants de limon glaciaire. Il aurait fallu sonder profondément pour le vérifier.

Il y avait, au-dessus de la couche dépotoir, et séparé d'elle par un remblai relativement stérile de 20 à $30 \mathrm{~cm}$, un niveau épais, assez riche, très probablement d'époque claudienne, autant qu'on puisse le dire au vu de la petite quantité de matériel qu'il a été possible d'examiner (quelques fragments de céramiques à parois fines sablées).

Notre intervention a dû se limiter à l'extraction de la plus grande quantité possible de matériel qui, pour gagner du temps, a été mis en sac, et le mélange argile-tessons a été ensuite lavé. Le mobilier, regroupé avec celui que nous a remis $R$. Pelletier, ne représente au total que quelques kilogrammes de tessons.

Les observations archéologiques se limitent donc à ces quelques lignes : pas de photographies, pas de relevés, pas de prise de niveau, pas de sondages complémentaires ni d'élargissement : on ne peut que le regretter au regard de l'importance de la découverte, mais cette faiblesse, assez fréquente à Lyon à cette époque, a été aggravée par les circonstances particulières de l'opération. Quoi qu'il en soit, l'étude devra se limiter au mobilier recueilli dont on ne peut donc dire dans quelle partie de l'atelier il était situé, s'il provient d'un sol de travail ou d'un dépotoir, quelle proportion de la production il peut représenter. La carence stratigraphique interdit en effet toute autre approche que typologique pour une datation. Il serait en outre étonnant que toute la production soit représentée sur une aussi faible surface et par un échantillonnage aussi réduit. On peut supposer que l'atelier a fabriqué d'autres types de céramique, ou des variantes des types représentés, et si nous insistons sur ce point, c'est pour éviter que Loyasse ne soit trop hâtivement interprété comme un petit atelier à faible production et à faible diffusion.

Le matériel recueilli n'évoque pas a priori des rebuts de fabrication comme on a l'habitude de les observer : pas de surcuits noirs ou verts comme à la Muette, pas de pièces collées, pratiquement pas de tessons déformés à l'exception de deux ou trois fragments de gobelets d'Aco. Nous nous sommes même demandé s'il s'agissait bien d'un atelier, mais la composition même du dépôt (absence de céramique autre que les imitations de sigillée ou les céramiques à parois fines, absence de mobilier métallique ou osseux et surtout présence de fragments de tubulures, de cales et de boulettes d'argile) permet bien de trancher par l'affirmative.

Ce mobilier paraissait ingrat à étudier de prime abord. Tous les éléments sont fragmentés à l'extrême, comme s'ils avaient été piétinés. Nous n'avons pu faire pratiquement aucun collage et de ce fait aucun profil complet n'a pu être relevé. Les parois minces sont réduites à l'état de fragments de la taille d'un ongle. Le mobilier peut être classé en plusieurs groupes :

- accessoires de fabrication : fragments de cales, de tubulures, boulettes d'argile plus ou moins cuites;

- imitations de sigillée : quelques centaines de fragments identifiables, rebords, pieds essentiellement. Une estampille a été recueillie par R. Pelletier, mais dans un autre secteur de la masse 4 du cimetière. Son appartenance aux productions de l'atelier a été contrôlée par analyse ;

- céramiques à parois fines lisses : 1901 fragments;

- gobelets d'Aco : 3831 fragments, dont quelques-uns déformés à la cuisson ainsi que quatorze tessons portant une glaçure plombifère vert jaunâtre qui correspondent également à des gobelets d'Aco;

- céramiques à parois fines à décor peigné : 78 tessons de petits vases globulaires ou bols. 
Quand il a été question de reprendre l'étude de cet atelier pratiquement inédit nous avons décidé de nous limiter strictement dans une première étape à l'inventaire analytique du mobilier recueilli en 1970. Dans la mesure où il est peu abondant, il était tentant de chercher à élargir notre connaissance de ces productions en étudiant le mobilier des sites de consommation, lyonnais ou extérieurs en particulier les tessons portant des estampilles. Cela aurait nécessité un important programme d'analyses et, de fait, retardé la publication. Ce travail étant destiné entre autres à permettre une identification précise de ces productions, nous avons recherché la précision dans la description des éléments caractéristiques.

J. L.

\section{LES PRODUCTIONS}

Le mobilier comprend 6066 tessons qui se répartissent, à l'exception de deux d'entre eux, en deux grandes catégories, imitations de sigillée et céramique à parois fines. L'histogramme effectué sur l'ensemble des tessons avant recollage montre une très nette prédominance des vases à parois fines qui représentent à eux seuls $96 \%$ du lot avec 5824 fragments (fig. 5).

\begin{tabular}{|l|c|}
\hline \multicolumn{1}{|c|}{ CATÉGORIES } & FRAGMENTS \\
\hline imitations T. S. & 240 \\
\hline parois fines & 5824 \\
\hline autres & 2 \\
\hline total & 6066 \\
\hline
\end{tabular}

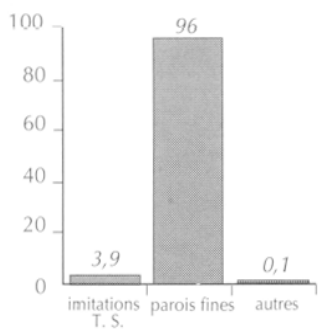

Fig. 5. Représentation respective des trois catégories de céramique.

LES IMITATIONS DE SIGILLEE (pl. 1 à 3)

\section{Définition}

Ce terme désigne des céramiques à pâte calcaire et vernis non grésé (rouge ou noir) cuites selon le mode $\mathrm{A}$ (Picon, 1973) reprenant plus ou moins librement des formes inspirées du répertoire italique précoce. Certains auteurs parlent de "pré-sigillées ", d'autres privilégient le terme d'imitations de Campanienne. Le choix de telle ou telle dénomination ne saurait, de toute façon, être totale- ment adéquat dans la mesure où il reste difficile de faire la part entre les influences de la céramique étrusco-campanienne et de la sigillée arétine, des filiations existant entre les deux répertoires.

\section{Typologie}

Les deux cent quarante fragments d'imitations se réfèrent à trois groupes morphologiques inégalement représentés sur le plan numérique : bols hémisphériques, plats, assiettes et bols à bord oblique, plats, assiettes et bols apparentés au service I. Seules deux formes ne s'intègrent pas dans l'un ou l'autre groupe : la première est une coupe à bord non profilé, de profil concave, sans doute à rapprocher des coupes Goudineau 3 (pl. 1, 1); la seconde est figurée par un fragment d'anse rubanée de type indéterminé (pl. 1, 2).

\section{LES BOLS HÉMISPHÉRIQUES (pl. 1, 3-4)}

Ils sont nettement minoritaires avec quatre exemplaires seulement. Ces formes qui évoquent les bols de type Lamboglia 1 en céramique campanienne B sont attestées à Lyon, sur les sites du Verbe-Incarné et de la rue des Farges, à l'époque augustéenne précoce, bien qu'en proportions négligeables en regard des autres types d'imitations de sigillée (Desbat et alii, 1989). Il faut par ailleurs souligner que les exemplaires connus sur ces deux sites très proches sont à vernis brun noir pour la plupart, ce qui ne semble pas se traduire dans notre matériel; les fragments de bols ayant conservé quelques traces de vernis présentent des teintes claires, orange à rouge.

\section{LES ASSIETTES ET LES BOLS À BORD OBLIQUE (pl. 1, 5-13)}

Ce groupe compte soixante-seize exemplaires dont l'état de fragmentation interdit de donner les proportions exactes de plats, assiettes et bols. On notera du moins que les assiettes dont on a pu mesurer les diamètres d'ouverture s'avèrent de dimensions modestes puisque tous ces diamètres sont compris entre 9 et $21 \mathrm{~cm}$. Il semble donc y avoir une majorité d'assiettes aux côtés desquels figurent seulement quatre ou cinq bols proprement dits (pl. 1, 12-13).

Les surfaces des vases, quand elles n'ont pas perdu toute trace de vernis, présentent des teintes claires. Ces formes issues du répertoire italique précoce reprennent de façon plus ou moins fidèle les types archaïques 
Goudineau 1 et 2 de la sigillée italique, eux-mêmes dérivés du type campanien Lamboglia 5/7. Elles constituent sans doute les témoins les plus probants des filiations ayant existé entre ces deux productions à la période préaugustéenne.

\section{LES FORMES APPARENTÉES AU SERVICE I (pl. 2 et 3)}

Ce troisième groupe correspond en fait à un conglomérat de vases qui s'apparentent de manière plus ou moins évidente selon les cas aux formes précoces du service I. Il se compose de vingt-deux assiettes et de six bols.

Six assiettes n'évoquent aucun des types connus en imitations ou bien en sigillée proprement dite. Une assiette (pl. 2, 1) se caractérise par une lèvre en bourrelet dégagée à l'intérieur par une gorge très large et prolongée par une paroi arrondie de profil évasé; ce type de bord, pour lequel on ne peut établir le moindre parallèle, que ce soit à Lyon, sur le limes, en Italie ou au Magdalensberg, figure en un seul exemplaire dans le mobilier de Loyasse. Quatre autres assiettes (pl. 2, 2-4) présentent une paroi nettement incurvée se terminant par une lèvre éversée soulignée à son extrémité supérieure par une fine rainure. De telles caractéristiques ne se retrouvent pas sur les types archaïques et précoces de la production arétine; par ailleurs, on constate l'absence totale de profils similaires, ou même approchants, dans le mobilier augustéen lyonnais.

Dix-sept assiettes peuvent, en revanche, être apparentées au service $I$. Une première série de quatre exemplaires (pl. 2, 5-8) se distingue par une lèvre débordante de section triangulaire, très nettement détachée de la paroi, qui rappelle les profils archaïques du service IA. Des formes comparables ont été produites en imitations par les ateliers de Bram (Passelac, 1986, pl. 1, n 140) et de Saint-Romain-en-Gal (Desbat, Savay-Guerraz, 1986, pl. $\left.2, n^{\circ} 2\right)$. Un exemplaire de Bram possède une carène à la liaison bord/fond que l'on retrouve sur l'assiette 5 (Passelac, 1986, pl. 1, n 70). On peut également signaler quelques parallèles avec des vases sigillés de Neuss (Ettlinger, 1983, pl. 9, $\mathrm{n}^{\circ}$ 17) et du Magdalensberg (Schindler, Scheffenegger, 1977, pl. 7, $\mathrm{n}^{\circ} 12$ ). Une deuxième série de treize assiettes (pl. 2, 9-10 et pl. 3, 1-11) offre un aspect moins homogène. Les lèvres, qu'elles soient triangulaires ou arrondies, nettement débordantes ou rapprochées de la paroi, sont néanmoins toutes soulignées par une rainure à leur extrémité supérieure. Certaines évoquent encore les profils du service IA (pl. 2,
9-10 et pl. 3, 1-2), d'autres se rapprochent davantage du service IB ou du moins des formes précoces de ce service (pl. 3, 3-11). Les bols, en particulier, présentent des lèvres en cloche directement inspirées des modèles sigillés (pl. 3, 12-16). On connaît des profils tout à fait comparables en sigillée sur différents sites, et notamment à Bâle où ils sont simplement définis comme formes précoces du service I (Fürger-Gunti, 1979). Les analogies que l'on peut donc relever avec la céramique sigillée précoce des principaux sites connus à l'époque augustéenne entérinent bel et bien un phénomène d'imitation perceptible mais plus difficile à cerner dans les groupes de formes archaïques. Une seule estampille, incomplète de surcroît s'ajoute aux productions de l'atelier : ARRE (...), (cf. infra, pl. 83, 1). Il s'agit probablement de l'adjectif dérivé du nom d'Arrezo.

M. G.

\section{LES CÉRAMIQUES À PAROIS FINES}

(fig. 6 et pl. 4 à 8 )

Elles comprennent 5824 tessons qui se rapportent à trois groupes de vases à pâte siliceuse cuite en mode A : vases lisses (pl. 4), vases guillochés (pl. 5, 1-12), gobelets d'Aco (pl. 5, 13-19 et pl. 6 à 8).

\section{LES VASES LISSES (pl. 4)}

Cent soixante-treize rebords et environ quatre-vingt fragments de fonds composent cet échantillon pour lequel aucun profil complet n'a pu être reconstitué. L'état de fragmentation du mobilier n'en a pas facilité l'analyse typologique mais l'examen détaillé des principaux éléments d'identification (bords, fonds, carènes) montre d'une part un répertoire typologique restreint, d'autre part un important déséquilibre numérique entre les types reconnus.

\begin{tabular}{|l|c|}
\hline \multicolumn{1}{|c|}{ GROUPES } & FRAGMENTS \\
\hline vases lisses & 1901 \\
\hline vases guillochés & 78 \\
\hline gobelets d'Aco & 3845 \\
\hline total & 5824 \\
\hline
\end{tabular}

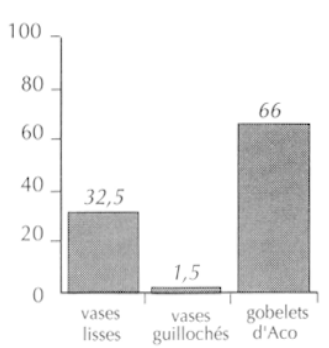

Fig. 6. Représentation respective des trois groupes de vases à parois fines. 
Ce lot comprend tout d'abord treize gobelets qui se rattachent à deux grandes séries attestées sous Auguste :

- les gobelets à bord concave (9 ex.) dont on a figuré les principales variantes, bord haut et très concave ( $\mathrm{pl} .4$, 1), bord court et faiblement concave (pl. 4, 2).

- quatre gobelets à bord lisse (pl. 4, 3-4) pourraient s'apparenter à certaines formes décorées présentes à Dangstetten (Fingerlin, 1986, 42-11 et 211-63) ; cependant l'absence totale de tessons décorés de pâte et d'aspect semblables ne permet pas de confirmer cette analogie. Il n'est d'ailleurs pas impossible que ce type de gobelets ait été, comme les gobelets à bord concave, décoré ou non suivant les cas.

Deux dernières formes ne s'intègrent pas exactement dans l'une ou l'autre série : le rebord 5 ćvoque davantage les gobelets-tonneaux produits à la Muette. Le gobelet 6 qui se caractérise par une lèvre en bourrelet et un diamètre inhabituel n'appartient pas à un type connu.

Aux côtés de cet échantillon à la fois restreint en nombre et dispersé sur le plan typologique, on trouve un ensemble de trois cent sept tessons se rapportant vraisemblablement au même type de gobelets cylindriques à fonds moulurés dont la production sera encore attestée sur l'atelier de la Muette. Cet ensemble comprend cent quarante-quatre fragments de fonds moulurés et de panses carénées (pl. 4, 12-17) d'identification certaine, mais sans doute aussi tout ou partie des cent soixante-trois bords simples non profilés d'aspect similaire (pl. 4, 7-11); l'absence des fonds caractéristiques des gobelets tronconiques ou des bols hémisphériques limite, en effet, en grande partie les risques d'erreur. Il convient de souligner l'identité quasi parfaite entre les gobelets cylindriques de Loyasse et ceux que l'on retrouve dans les productions de la Muette. Il s'agit d'un type bien défini dont les traits morphologiques (profils des différents éléments du vase mais aussi rapports dimensionnels) ne varient pas d'un atelier à l'autre. Mais on peut aussi faire les mêmes remarques à propos de gobelets cylindriques mis au jour en Italie, en Gaule, comme à l'est ou au nord de l'Europe. Les contextes de datation indiquent une chronologie haute mais on dispose rarement d'ensembles de référence numériquement probants. Ces gobelets ne semblent pas avoir connu de production et de diffusion massives, que ce soit à partir de Lyon ou d'autres centres producteurs. Des fragments de fonds attestent d'autres vases lisses de types indéterminés (pl. 4, 18-21).

M. G.
LES VASES À DÉCOR GUILLOCHÉ OU PEIGNÉ (pl. 5, 1-12)

Ce dernier groupe peu représenté au sein du mobilier de Loyasse (78 tessons c'est-à-dire 1,5\% des céramiques à parois fines) comprend lui aussi des vases à pâte siliceuse mais qui s'éloignent radicalement des séries précédentes par l'aspect des surfaces (teintes grises à brunes), la présence de décors guillochés, parfois striés ainsi que par une moindre qualité d'exécution.

Les cinq profils dessinés rendent compte de deux séries morphologiques bien distinctes : la première comporte des gobelets à bord non profilé et panse ovoïde guillochée ou ondée (pl. 5, 1-3) et la seconde des bols hémisphériques à bord simple vraisemblablement ornés de guillochis sur toute leur hauteur (pl. 5, 4-5).

Il s'agit là d'un groupe isolé dans le matériel de l'atelier, inconnu à la Muette et apparemment unique à Lyon si l'on en juge par l'absence totale de productions identiques voire comparables dans les contextes augustéens précoces et classiques du Verbe-Incarné et de la rue des Farges. Le site de la rue des Farges a d'ailleurs livré quelques exemplaires de gobelets à panse ovoïde offrant divers profils et ne formant pas, en tout état de cause, un type homogène. Ces vases, rassemblés dans le type IV de C. Grataloup, ont été un peu rapidement sans doute assimilés à ceux que nous présentons ici et de ce fait intronisés comme "productions probables de l'atelier de Loyasse et de la Muette".

En dernier lieu, quatre fonds de bols ou de gobelets et trois fragments isolés (pl. 5, 6-12) déterminent quelques vases peignés. Ce type de vases est attesté à Lyon sous Auguste (Verbe-Incarné, rue des Farges) bien que toujours en proportions très restreintes.

Le mobilier de Loyasse, en dépit de sa faiblesse numérique et de sa fragmentation, offre indéniablement un faciès ancien. On connaît sur le limes et à Lyon quelques ensembles de référence compris entre les années 40 avant J.-C. et 15-10 avant J.-C. Les datations en sont essentiellement fondées sur les indices de fréquence respectifs de trois grands groupes de vases sigillés : formes archaïques et précoces, service I, service II. Si l'on raisonne de la même façon sur les imitations de l'atelier, on admettra d'emblée une date antérieure à l'horizon Dangstetten : absence du service II, absence du service IC et forte proportion de types archaiqques et précoces.

Les formes archaïques sont ici représentées par les bols hémisphériques et les bols et assiettes à bord oblique, types caractéristiques à Lyon des premières occupations 
urbaines (Desbat et alii, 1989). Le groupe des vases " apparentés au service I " constitue un chapelet de formes rares ou inédites dans nos contextes de référence; il comprend en effet une majorité de types inconnus ainsi quc des vases rappelant les profils du service IA. On note cependant la présence de quelques rebords assimilables au service IB ou du moins aux formes précoces de ce service qui pourraient constituer les éléments les plus récents du lot.

Le problème est de savoir si l'on considère que ces vases sont autant de variantes d'un service déjà fixé ou bien qu'ils correspondent à une période où la diversité des profils rend compte de l'absence de toute standardisation dans la production sigillée. De fait, les comparaisons que l'on a pu établir avec le matériel des ateliers de Saint-Romain-en-Gal ou de Bram ainsi qu'avec des formes précoces de Neuss, du Magdalensberg ou de Bâle mettent en évidence plus de ressemblances à proprement parler que de correspondances typologiques précises.

On a donc tout lieu de penser que les imitations de l'atelier de Loyasse s'inscrivent dans un horizon chronologique ancien 40-20 avant J.-C. Elles forment néanmoins un ensemble particulier dont on ne retrouve pas les éléments les plus originaux dans le mobilier des premières occupations du Verbe-Incarné et dont on ne peut apprécier la réelle valeur représentative.

De telles circonstances pourraient naturellement s'expliquer par un décalage chronologique plus ou moins important mais ne doit pas négliger non plus la nature même des sites concernés : il existe, en effet, très probablement, des différences sensibles dans la composition d'ensembles strictement contemporains entre sites de production et sites de consommation.

M. G.

LES GOBELETS D’ACo (pl. 5, 13-19 et pl. 6 à 8)

\section{Forme}

On connaît essentiellement deux formes pour ce type de gobelets : une forme haute tronconique resserrée vers l'ouverture avec une base plus étroite. Le diamètre maximum se situe au tiers supérieur et le rapport hauteur-largeur est de l'ordre de 3 pour 1 . Il existe une forme basse, de même structure, mais dont le rapport hauteur-largeur est de 1 pour 1. Présente à la Muette, elle ne semble pas avoir été fabriquée à Loyasse. Une seule variante de la forme haute classique est représentée : alors que tous les rebords sont bombés et terminés par une très fine lèvre rabattue vers l'extérieur, un fragment présente au-dessus de la frise, et légèrement en retrait, un rebord vertical non bombé, et sans aucune lèvre. Il s'agit d'un exemplaire unique qui, vraisemblablement illustre plus une fantaisie d'un potier qu'il ne représente un type différent produit en série.

\section{Décor}

Un seul type de décor est présent dans l'échantillonnage étudié à Loyasse, et c'est le plus simple, on peut même dire le plus classique : un semis de picots (comaregen dans la littérature allemande) qui occupe les deux tiers inférieurs de la panse, celle qui est réalisée au moule, le tiers supérieur, en bandeau lisse légèrement bombé et sommé d'une petite lèvre étant réalisé au tour après moulage de la partie inférieure. En bas, le semis de picots laisse libre des zones triangulaires, pointes en haut, dont certaines sont timbrées d'un motif. La zone décorée de picots est limitée en haut par une frise végétale. Au contact de cette frise, le semis de picots réserve un espace pour la signature, presque toujours encadrée de dauphins.

Le semis de picots ne présente pas de caractéristiques particulières. Les picots sont de formes et de dimensions très variables, certains étroits avec des angles arrondis, d'autres plus courts et plus larges avec des arêtes très nettes. Leur implantation est variable, le semis étant plus ou moins dense, mais il reste très régulier et se resserre vers le bas du gobelet. On peut simplement observer à Loyasse une tendance nette à l'utilisation de picots de petites dimensions, mais disposés de manière très serrée. Au-delà de l'anecdote, ces observations étant naturellement trop superficielles pour identifier telle ou telle production, reste posé le problème de la réalisation des moules : la mise en place de ces décors en creux dans des troncs de cône extrêmement étroits, et avec une régularité parfaite, ne peut s'expliquer seulement par la virtuosité du potier. Mais si un semis de picots ne peut permettre d'identifier un atelier, on peut supposer que les éléments figurés, frises, graphies de signatures, motifs d'accompagnement sont plus caractéristiques. C'est la raison pour laquelle nous serons le plus précis possible dans la description, tout en essayant d'éviter le langage sophistiqué (mais parfois poétique) de l'abbé Hermet. 


\section{Les frises (pl. 6)}

Elles semblent toujours encadrées par deux filets en relief, mais, dans quelques cas, le filet supérieur a disparu, effacé lors du tournage du rebord lisse hors du moule, ou plutôt à l'occasion des lissages de finition après démoulage.

Les quinze frises de Loyasse actuellement connues sont toutes constituées d'alignements d'éléments végétaux plus ou moins stylisés et imbriqués. Mais, et c'est pour l'instant une originalité de cet atelier, certaines frises sont utilisées tantôt seules (frises simples), tantôt surmontées d'une ligne de postes (frises composées).

1. Frise simple : deux feuilles orientées à gauche, symétriques par rapport à une tige horizontale qui touche le groupe précédent. À la base des feuilles, deux petites feuilles également symétriques; largeur de filet à filet : $8 \mathrm{~mm}$ à $8,5 \mathrm{~mm}$.

2. Frise composée : même frise que le $n^{\circ} 1$, surmontée d'une ligne de postes; largeur : $10 \mathrm{~mm}$.

3. Frise composée : deux feuilles très étroites, orientées à gauche, dont les extrémités évidées sont enroulées en crosse, et symétriques par rapport à une tige axiale plus courte, arrondie et nervurée. La ligne végétale est surmontée d'une frise de postes; largeur : 11,5 mm.

4. Frise simple : deux feuilles orientées à gauche, symétriques très arrondies. Elles se réunissent en pointe horizontale, parfois déplacée vers le haut; intérieur des feuilles très creusé ; largeur : 8,5 à $9 \mathrm{~mm}$.

5. Frise composée : même ligne végétale que le $n^{\circ} 4$, surmontée d'une frise de postes; largeur : 11,5 mm.

6. Frise simple : deux feuilles allongées, orientées à gauche, très creusées à l'intérieur; type comparable au $\mathrm{n}^{\circ} 4$, mais plus long; l'imbrication des feuilles est très irrégulière ; largeur : $8 \mathrm{~mm}$.

7. Frise simple : feuilles symétriques par rapport à l'axe de la frise, très minces, à extrémité élargie, retroussée et creusée. Les paires sont groupées par dix et par l'extrémité des feuilles pour chaque groupe de cinq; largeur : $9 \mathrm{~mm}$. Certains exemplaires, très empâtés, donnent l'impression d'être plus larges, mais il s'agit bien en fait du même poinçon.

8. Frise simple : comparable au $n^{\circ} 3$, mais plus stylisée. Deux tiges, orientées à gauche, symétriques par rapport à l'axe de la frise, les extrémités rejoignent deux lignes de cercles et s'y lient à un point sur deux. Entre deux tiges, petits éléments plus larges et plus creusés; largeur : $7 \mathrm{~mm}$.

9. Frise composée : en haut, frise de petits éléments très stylisés, très serrés. Au-dessous, ligne de couronnes ouvertes vers le haut; largeur : $8 \mathrm{~mm}$. C'est de loin la frise la plus fréquente.

10. Frise composée : type très comparable au $n^{\circ} 9$; en bas, même ligne de couronnes; au-dessus, les éléments végétaux sont remplacés par une frise de postes; largeur : $8 \mathrm{~mm}$.

11. Frise simple très élégante : larges feuilles orientées à droite, arrondies et creusées, encadrant un motif triangulaire dont la pointe touche la jonction des feuilles précédentes. Entre chaque paire de feuilles, et de chaque côté du motif central, deux petits cercles; largeur : $7 \mathrm{~mm}$.

12. Frise composée : en bas, deux feuilles orientées à gauche, genre feuilles de gui, symétriques et évidées. Au-dessus, frise de postes; largeur : $8 \mathrm{~mm}$.

13. Frise composée : en bas, deux très petites feuilles orientées à droite, symétriques, presque rondes et évidées. En haut, frise de postes; largeur estimée : 7,5 mm.

14. Frise composée : en bas, deux petites feuilles allongées, symétriques, orientées à gauche. Entre elles, deux baguettes élargies en haut. Au-dessus, ligne de postes; largeur : $6,5 \mathrm{~mm}$. La partie inférieure de cette frise, une des plus fines de l'atelier, est comparable à certaines frises de Chrysippvs de la Muette.

15. Frise simple : deux baguettes symétriques, orientées à droite, torsadées, se terminent par un cercle. Entre les baguettes, petits motifs allongés à extrémité creusée; largeur : $7 \mathrm{~mm}$. Elle peut, comme le $\mathrm{n}^{\circ} 14$, être comparée à une des frises utilisées à la Muette mais par Hilarvs Aco.

\section{Bas du décor (pl. 7, 1-5)}

Les zones triangulaires du bas des gobelets sont, dans la plupart des cas, timbrées d'un petit motif :

- une larme, ou fleur en bouton, doublée à l'intérieur, et surmontée d'un anneau pointé à l'intérieur (pl. 7, 2-3) . Deux poinçons ont été utilisés, donnant un motif de $5,5 \mathrm{~mm}$ de haut dans un cas, de 7,5 $\mathrm{mm}$ dans l'autre;

- plus rare, un cercle cranté, peut-être une couronne stylisée $(5 \mathrm{~mm})(\mathrm{pl} .7,4)$; 
- un seul fragment porte une petite fleur stylisée à quatre pétales, de forme sensiblement carrée, avec un point au centre $(4 \times 4 \mathrm{~mm})(\mathrm{pl} .7,5)$.

\section{Les signatures (pl. 7, 6-16)}

Comme la plupart des gobelets de type ACO à semis de picots, la signature est placée sous la frise, dans un espace laissé libre par les picots. À Loyasse, elle est presque toujours encadrée par deux dauphins affrontés.

Vingt-huit tessons portent des fragments de signature; aucune n'est complète, mais il est cependant aisé de reconstituer un premier type : HILARVS ACO; ces noms sont extrêmement répandus, soit seuls, soit associés. Hilarvs Aco est connu en particulier à la Muette, où sa production, moins variée que celle de Chrysippvs, est cependant très abondante. Cette signature n'a pas été appliquée dans le moule avec un seul poinçon par nom ou un seul pour les deux. Elle n'a pas été non plus tracée à la pointe, mais composée dans chaque moule avec un poinçon par lettre. Certaines d'entre elles présentent en effet des détails caractéristiques que l'on retrouve sur des tessons issus de moules différents.

$\mathrm{L}$ : la barre horizontale prend naissance au-dessus du bas de la barre verticale; elle est de ce fait inclinée.

A : barre horizontale brisée vers le bas. Un A du même type, mais d'un poinçon différent, est aussi utilisé à la Muette.

$\mathrm{V}$ : les deux hastes s'épaississent vers le haut et se touchent à peine en bas.

Toutes les hastes comportent un apex au sommet et un empattement à la base. HILARVS et ACO sont toujours séparés par un picot isolé.

Le deuxième type de signature reste incomplet; il n'est représenté que par deux fragments et ne comporte pas, semble-t-il, de dauphin d'encadrement. Le semis de picots cerne étroitement à gauche un A qui est le même que celui d'HILARVS ACO. On pense, bien sûr, à la première lettre de ACO. Il y aurait alors trois solutions :

- ou bien ACO est seul;

- ou bien HILARVS (ou un autre nom) et ACO sont séparés;

- ou enfin, ACO précède un autre nom (HILARVS, DIOPHANES, etc.), il est impossible de trancher pour l'instant entre ces trois hypothèses. Un de ces deux fragments porte la frise $n^{\circ} 7$.
Les dauphins (pl. 8)

Ils font partie intégrante de la signature HILARVS ACO et lui servent d'encadrement, mais comme nous n'avons pu reconstituer aucune signature complète, nous n'avons pas de paire complète de dauphins. Nous pouvons seulement constater qu'à l'intérieur des deux séries, à gauche et à droite, ils sont très différents par leurs dimensions et leurs détails, ainsi que par leur style qui reste, en général, fort médiocre. Chacun des types est représenté par plusieurs fragments.

\section{Série de gauche (pl. 8, 1-3)}

- Dauphin G1 : queue platc, grossièrement incisée au bout; corps raide, la jonction corps/queue se faisant en angle : c'est le détail le plus caractéristique du poinçon. Pas de nageoire ventrale.

La nageoire dorsale est figurée par un point à la jonction des parties avant et arrière du corps. Une autre nageoire dorsale très arrondie vient mourir sur le dessus de la tête ; gueule ouverte; œil peu marqué ou inexistant. Longueur estimée à 16 ou $17 \mathrm{~mm}$.

- Dauphin G2 : encore plus fruste que le précédent. Le corps est anguilliforme, le relief accentué. Forme générale en arc de cercle, queue fortement barbelée de quatre incisions. À la jonction queue/corps, nageoires ventrales et dorsales sont détachées par une forte incision, la nageoire dorsale figurée par des épines inclinées vers l'avant, plantées sur la tête. La gueule est ouverte, la mâchoire supérieure allongée; pas d'œil (?); longueur estimée à $13 \mathrm{~mm}$.

- Dauphin G3 : proche de G1 mais d'un style très supérieur, il manque la queue; la jonction queue/corps est plus souple, pas de nageoire ventrale à mi-corps, mais la dorsale est bien plus développée. La tête, très large, porte une nageoire à six épines très fine. Gueule ouverte à mâchoire nervurée. Eil très dessiné, avec ses «paupières »; longueur estimée à $16 \mathrm{~mm}$.

\section{Série de droite (pl. 8, 4-5)}

- Dauphin D1 : grand dauphin de très bonne qualité; manque l'extrémité du museau. Mouvement très souple; queue très fourchue et légèrement barbelée. La partie arrière du corps présente un étranglement qui est peutêtre un accident. À mi-corps, les nageoires dorsales et vcn- 
trales sont bien dessinées. Sur la tête, nageoire très inclinée vers l'arrière : longueur estimée à $20 \mathrm{~mm}^{7}$.

- Dauphin D2 : partie avant semblable à D1, mais il se termine par une queue fourchue très courte, (disproportionnée par rapport au reste du corps), précisément au point où nous avons cru discerner une cassure du poinçon Dl, qui aurait été cassé et retouché : longueur $17 \mathrm{~mm}$.

- Dauphin D3 : très net, mais de mauvaise facture, proche du poinçon G2. Corps en arc de cercle très ouvert, queue fourchue et non incisée; deux nageoires étroites à mi-corps, avec épines. Tête à gueule entrouverte, mâchoire supérieure proéminente. (Eil très empâté; longueur $16 \mathrm{~mm}$.

\section{CONCLUSION}

Il convient de rappeler au préalable les problèmes que pose la nature même de ce gisement. Si la découverte isolée d'une seule fosse contenant des rebuts de cuisson atteste bel et bien la présence d'un atelier, elle ne nous donne en revanche aucun renseignement précis sur le volume et la diversité de la production, sur l'organisation de l'atelier et son rôle dans l'agglomération antique. On doit se poser la question de la valeur représentative du mobilier de cette fosse. Les productions d'imitations de sigillée, de céramiques à parois fines lisses ou guillochées et de gobelets d'Aco étaient-elles associées à d'autres productions, ou les potiers s'étaient-ils spécialisés dans ce registre-là?

D'autre part, estimer l'importance de cet atelier en examinant le matériel de sites de consommation reste à l'heure actuelle une entreprise difficile : il existe en effet, à l'évidence, des différences sensibles dans la composition d'ensembles " contemporains ", au sens large, entre sites de production et sites de consommation.

Enfin, l'élaboration d'une véritable étude de diffusion de ces productions supposerait que l'on puisse identifier et certifier l'origine des vases, qu'ils proviennent de sites de consommation locaux, régionaux, ou bien de sites éloignés. De telles conditions ne sont pas réunies dans l'état actuel de la recherche : les analyses physico-chimiques effectuées sur un échantillon d'imitations provenant des sites du Verbe-Incarné et de la rue des Farges

7. Le seul tesson présentant ce motif complet a été égaré depuis 1970: il ne figure donc pas dans l'illustration. révèlent l'existence de compositions proches mais non identiques, dont la diversité pourrait indiquer l'existence de plusieurs "ateliers" ou points de production possibles dans le même secteur.

J. L.

\section{LA CHRONOLOGIE DE L'ATELIER}

Les comparaisons établies avec les premiers contextes lyonnais du Verbe-Incarné, qui appartiennent à l'occupation urbaine la plus ancienne de la colonie, apportent des renseignements de différents ordres et de valeurs inégales permettant néanmoins de circonscrire un horizon chronologique (tabl. II).

Tabl. II. Présence/absence des principaux types d'imitations dans les contextes anciens de Lyon. Verbe-Incarné (d'après Desbat et alii, 1989), dépôt L3 de la montée de Loỵasse (d'après (iènin, 1994).

\begin{tabular}{|l|c|c|c|}
\hline \multicolumn{1}{|c|}{ GROUPES } & V-I 1 & ATELIER & DÉPÔT L3 \\
\hline formes précoces & 0 & 0 & 0 \\
\hline service IA & 0 & 0 & 0 \\
\hline service IB & & 0 & 0 \\
\hline
\end{tabular}

Le mobilier de la première trame urbaine se caractérise en effet par un fort effectif de formes sigillées archaïques (Goudineau 1 et 2, formes du service IA) associées à un lot relativement important d'imitations à vernis non grésé et reprenant plus ou moins fidèlement les mêmes types de vases (Desbat et alii, 1989). Si on juxtapose ce matériel d'habitat et les imitations de sigillée de l'atelier, force est de constater qu'il n'y a pas adéquation " parfaite " entre les deux faciès, que l'on prenne ou non en compte le déséquilibre numérique entre les deux ensembles et la différence de nature de ces dépôts. Les formes archaïques, bien que très fortement représentées à Loyasse, y coexistent avec un petit nombre de vases qui s'apparentent davantage aux formes précoces du service IB proprement dit. Ces formes précoces qui sont totalement absentes au Verbe-Incarné, en sigillée et a fortiori en imitations de sigillée, semblent indiquer un léger décalage chronologique avec les premiers habitats lyonnais.

On peut en revanche relever plus de similitudes entre cet ensemble et celui particulièrement riche du dépôt augustéen L.3 de la montée de Loyasse. Ce dépôt consti- 
tue un point de repère particulièrement intéressant à plusieurs égards : il a en effet livré un demi-as de copia qui fournit d'emblée un terminus de 30 avant J.-C. et qui se trouve associé à un matériel céramique à la fois riche et diversifié (Genin, 1994).

Ce mobilier comprend notamment, au sein d'un nombre important de formes sigillées et d'imitations caractéristiques des années 40 avant J.-C. (peut-être en partie produites par les ateliers du groupe Loyasse), un lot de bols à vernis grésé montrant des profils précoces du service IB et qui dénotent clairement une évolution typochronologique par rapport au premier répertoire des sigillées italiques.

Les indices fournis par l'étude des vases à parois fines semblent aller dans le même sens (tabl. III). La céramique à parois fines du Verbe-Incarné se compose pour l'essentiel de gobelets à bord concave (décors de semis d'épines et de cordes) et de gobelets d'Aco. Ces derniers, également nombreux dans le matériel de l'atelier, y sont néanmoins associés à des types de vases lisses, et notamment un lot très important de gobelets cylindriques à pied mouluré, dont la fabrication va perdurer à Lyon sous Auguste aux ateliers de la Muette.

On a donc tout lieu de penser que le mobilier de l'atelier n'est pas strictement contemporain des premières occupations urbaines de Lyon, mais qu'il rend compte d'une période lègèrement plus récente, déjà représentée à Lyon par le dépôt L3, et correspondant selon toute vraisemblance aux années 30/20 avant J.-C. Dans l'état actuel de la recherche, nous pouvons donc admettre que l'atelier a fonctionné pendant cette période, ce qui ne veut pas dire que le début de la production ne puisse pas remonter à une époque légèrement antérieure, et que la production ne se soit pas poursuivie, comme à Saint-

Tabl. III. Présence/absence des principaux types de vases à parois fines dans les contextes anciens de Lyon. Verbe-Incarné (d'après Desbat et alii, 1989), dépôt L,3 de la montée de Loyasse (d'après Genin, 1994).

\begin{tabular}{|l|c|c|c|}
\hline \multicolumn{1}{|c|}{ GROUPES } & V-I 1 & ATELIER & DÉPÔT L3 \\
\hline gobelets à bord concave & O & 0 & 0 \\
\hline gobelets d'Aco & 0 & O & O \\
\hline gobelets cylindriques & & O & O \\
\hline gobelets-tonneaux & & & 0 \\
\hline
\end{tabular}

0 : types faiblement représentés $\quad \mathbf{O}$ : types fréquents
Romain-en-Gal, au moins jusqu'à la fin du règne d'Auguste.

Les rapports entre les ateliers de Loyasse et de la Muette n'ont jamais pu être clairement établis. L'étude menée conjointement sur ces deux ateliers démontre du moins qu'il n'y a pas un écart chronologique important entre la période d'activité représentée par la fosse de Loyasse et l'ensemble I de la Muette et que certains types de vases à parois fines sont communs aux deux répertoires. De plus, la signature HILARVS ACO, seule marque de gobelets d'Aco attestée à Loyasse, est également représentée à la Muette, et une frise supérieure pourrait être commune aux deux ateliers. Mais de tels indices ne traduisent pas obligatoirement une continuité entre les potiers de Loyasse et ceux de la Muette.

M. G.

\section{IMITATIONS, CONTREFAÇONS, SUCGURSALES OU TRANSFERT DE TECHNOLOGIE?}

Les fabriques d'imitations de sigillée sont traditionnellement considérées comme des fabriques indigènes tenues par des potiers qui réalisaient ce type de céramiques parce qu'ils ne maitrisaient pas les techniques requises pour la production de "vraies sigillées » : sélection d'argiles, mode de cuisson et par conséquent réalisation de fours complexes. Autant dire que la production d'imitations existait "par défaut ». Dans la même optique, on a longtemps pensé à Lyon que Loyasse rendait compte d'une phase initiale préparant le terrain à une "véritable " production sigillée représentée par les ateliers de la Muette. Cette façon de voir les choses traduit l'idée d'une évolution linéaire. Or, les recherches les plus récentes menées dans ce domaine montrent que la réalité n'est peut-être pas si simple.

À la question de l'identité des potiers et à l'argument selon lequel ces derniers fabriquaient des imitations uniquement parce qu'ils ne savaient pas faire de la sigillée, on peut objecter plusieurs remarques.

Les timbres de potiers sur imitations, bien que relativement rares à Lyon, ne reproduisent pas des noms à consonance gauloise, comme c'est le cas, par exemple, sur les productions de terra nigra du $\mathrm{I}^{\mathrm{Ir}} \mathrm{s}$. après J.-C. L'unique timbre qui se rattache stricto sensu à l'atelier de Loyasse, ARRE (TINVM), témoigne à lui seul de relations étroites avec la tradition italique; n'oublions pas cependant qu'on peut aussi le lire "à la manière de " ou " comme à Arezzo ». 
La production de céramiques à parois fines lisses, de gobelets d'Aco et de vases à glaçure plombifère atteste l'emploi et la maittrise parfaite de techniques nécessairement importées d'Italie, ce qui fragilise d'ailleurs, dans le même temps, l'hypothèse d'un atelier purement indigène.

La mise en parallèle d'ateliers aussi éloignés que ceux de Loyasse et de Bram (Passelac, 1986) fait ressortir un certain nombre de traits communs dans la typologie des vases, fait qui semble contredire l'existence de répertoires exclusivement locaux s'étant développés de façon autonome. La très forte influence du répertoire italique précoce a donc, en partie, donné lieu à la création de "variantes " similaires et parfois identiques d'une région à l'autre.

On sait aujourd'hui que des ateliers d'imitations se sont développés et ont fonctionné en Italie à la même époque que les ateliers de sigillée : cela tendrait également à prouver que cette production répond à une demande et n'est pas a priori un phénomène dépendant de contingences techniques. Le fait même qu'on ait continué à fabriquer des imitations jusqu'à la fin du règne d'Auguste, voire après, comme par exemple à Saint-Romain-en-Gal (Desbat, Savay-Guerraz, 1986), établit qu'il devait exister aussi une clientèle pour ce genre de vaisselle (question de goût ou question de prix?). Rien ne permet d'affirmer que la production d'imitations à Lyon se limite à la période d'activité de Loyasse (ou des officines locales révélées par les analyses de pâtes) et que d'autres ateliers n'aient pas poursuivi dans cette voie, parallèlement aux ateliers de sigillée, en reproduisant tardivement les formes les plus anciennes du répertoire précoce.

Le schéma qui fait de l'atelier de Loyasse un atelier indigène correspondant à une phase initiale de la production sigillée à Lyon peut donc aujourd'hui être sérieusement remis en cause, même si l'on ne peut encore répondre de façon définitive aux questions portant sur l'origine et le développement de ce type d'officines. Les résoudre ou approcher des solutions suppose que l'on avance dans deux directions : la reprise des fouilles, même très limitées, qui permettrait d'élargir l'échantillonnage des productions puisqu'on ne peut envisager de décapages extensifs destinés à connaître l'organisation de l'atelier, ainsi que la recherche systématique d'ensembles contemporains sur des lieux de consommation, à Lyon, mais surtout à l'extérieur. Leur étude, fondée en particulier sur des analyses physico-chimiques d'argiles, permettra de cerner la diffusion de ces productions. Ces deux axes de recherche complémentaires n'ont rien d'irréaliste à moyen terme.

M. G., J. L.

\section{ANALYSE DES PÂTES}

L'atelier de Loyasse a produit à la fois des « imitations " de sigillées et des céramiques à parois fines du type gobelets d'ACO : les premières ont été fabriquées en pâte calcaire $\left(\mathrm{m}_{\mathrm{CaO}}=20,3 \%\right)$ et les secondes en pâte non calcaire $\left(\mathrm{m}_{\mathrm{CaO}}=1,7 \%\right)$.

\section{LES « IMITATIONS » DE CÉRAMIQUES SIGILLÉES}

L'étude des compositions des céramiques à pâte calcaire de l'atelier de Loyasse a porté sur vingt-huit exemplaires d'imitations de sigillées. Le tableau IV donne la composition chimique moyenne et les écarts-types correspondants du groupe de référence de Loyasse.

Ce groupe de Loyasse et ceux de la Muette ont servi de référence pour rechercher l'origine d'un certain nombre de productions anciennes découvertes sur divers sites lyonnais et viennois. Très peu d'exemplaires peuvent être attribués indiscutablement à l'atelier de Loyasse ou à l'atelier de la Muette. Les ressemblances de compositions sont néanmoins importantes, les différences observées semblent être de l'ordre de celles que l'on connaît dans le complexe fluvio-glaciaire régional. Ces résultats per-

Tabl. IV. Composition chimique moyenne du groupe des imitations de céramiques sigillées de l'atelier de Loyasse.

Éléments majeurs en pourcentages d'oxydes

\begin{tabular}{|c|c|c|c|c|c|c|c|c|c|c|}
\hline $\begin{array}{c}\text { Loyasse } \\
(\mathbf{n}=28)\end{array}$ & $\mathrm{Na}_{2} \mathrm{O}$ & $\mathrm{K}_{2} \mathrm{O}$ & $\mathrm{MgO}$ & $\mathrm{CaO}$ & $\mathrm{MnO}$ & $\mathrm{Al}_{2} \mathrm{O}_{3}$ & $\mathrm{Fe}_{2} \mathrm{O}_{3}$ & $\mathrm{SiO}_{2}$ & $\mathrm{TiO}_{2}$ & $\mathrm{P}_{2} \mathrm{O}_{5}$ \\
\hline $\mathrm{m}$ & 0,8 & 2,1 & 1,6 & 20,3 & 0,112 & 11,9 & 4,7 & 57,4 & 0,51 & 0,4 \\
\hline$\sigma$ & 0,1 & 0,1 & 0,2 & 1,6 & 0,019 & 0,3 & 0,2 & 1,3 & 0,02 & 0,1 \\
\hline$\sigma \%$ & 9 & 4 & 12 & 8 & 17 & 2 & 5 & 2 & 4 & 33 \\
\hline
\end{tabular}

Éléments traces en PPM

\begin{tabular}{|c|r|r|r|r|r|r|r|r|r|r|}
\hline $\begin{array}{c}\text { Loyasse } \\
(\mathbf{n = 2 8})\end{array}$ & $\mathrm{Rb}$ & \multicolumn{1}{|c|}{$\mathrm{Sr}$} & $\mathrm{Ba}$ & $\mathrm{Ni}$ & $\mathrm{Zn}$ & $\mathrm{Cr}$ & $\mathrm{Zr}$ & La & $\mathrm{Ce}$ & $\mathrm{V}$ \\
\hline $\mathrm{m}$ & 101 & 305 & 804 & 55 & 77 & 64 & 168 & 18 & 75 & 72 \\
\hline$\sigma$ & 19 & 15 & 179 & 7 & 12 & 5 & 16 & 13 & 6 & 6 \\
\hline $\boldsymbol{\sigma} \%$ & 18 & 5 & 22 & 13 & 16 & 7 & 9 & 71 & 8 & 9 \\
\hline
\end{tabular}


mettent de supposer qu'il existe à Lyon, ou tout au moins dans sa proche région, d'autres ateliers encore inconnus.

Les compositions chimiques des céramiques à parois fines sont homogènes et ont des caractéristiques qui permettent de bien les distinguer des autres productions régionales de ce type et notamment des productions de la Muette et de Saint-Romain-en-Gal.
Dix exemplaires ont été analysés et seuls les huit constituants chimiques majeurs ont été mesurés. Le tableau $V$ résume les caractéristiques de ces productions.

A. S.

Tabl. V. Composition chimique moyenne du groupe des céramiques à parois fines de l'atelier de Loyasse.

Éléments majeurs en pourcentages d'oxydes

\begin{tabular}{|c|c|c|c|c|c|c|c|c|}
\hline $\begin{array}{c}\text { Loyasse } \\
(\mathrm{n}=10)\end{array}$ & $\mathrm{K}_{2} \mathrm{O}$ & $\mathrm{MgO}$ & $\mathrm{CaO}$ & $\mathrm{MnO}$ & $\mathrm{Al}_{2} \mathrm{O}_{3}$ & $\mathrm{Fe}_{2} \mathrm{O}_{3}$ & $\mathrm{SiO}_{2}$ & $\mathrm{TiO}_{2}$ \\
\hline $\mathrm{m}$ & 1,9 & 1,2 & 1,7 & 0,032 & 18,2 & 6,3 & 69,7 & 0,93 \\
\hline$\sigma$ & 0,3 & 0,2 & 0,5 & 0,002 & 1,5 & 0,5 & 2,4 & 0,06 \\
\hline$\sigma \%$ & 6 & 14 & 32 & 8 & 8 & 9 & 4 & 6 \\
\hline
\end{tabular}



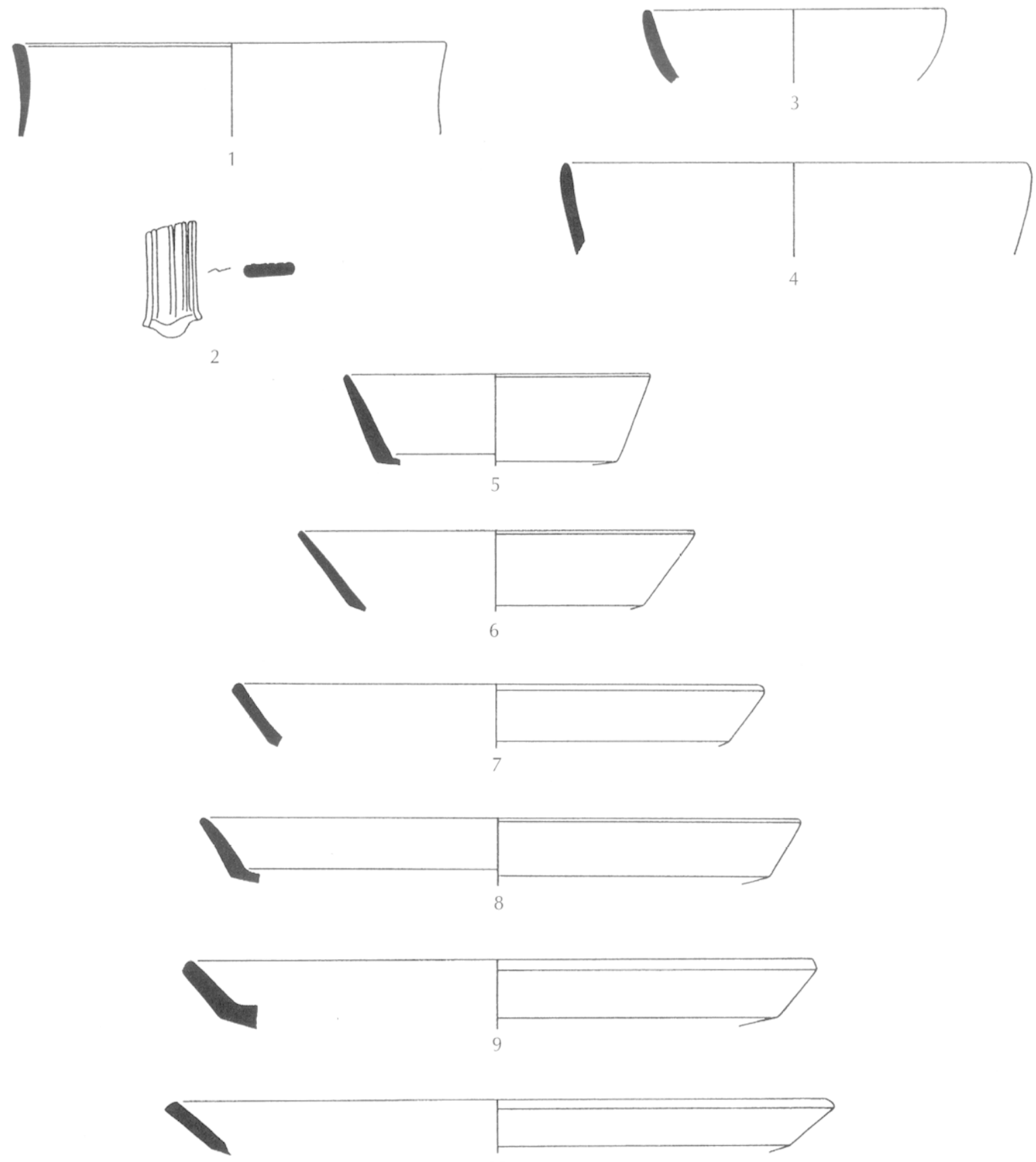

10

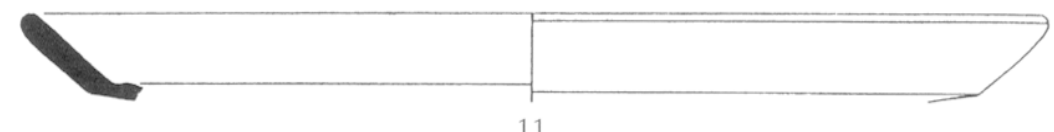

11

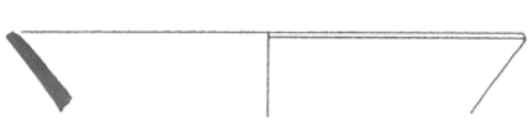

12

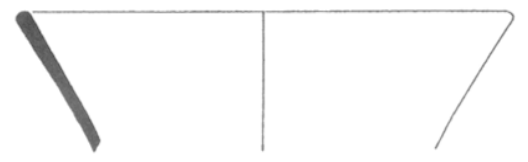

13

Pl. 1. Imitations de sigillée: formes uniques (1-2), bols hémisphériques (3-4), coupes et assiettes à bord oblique (7-13) (échelle: 1/2). 

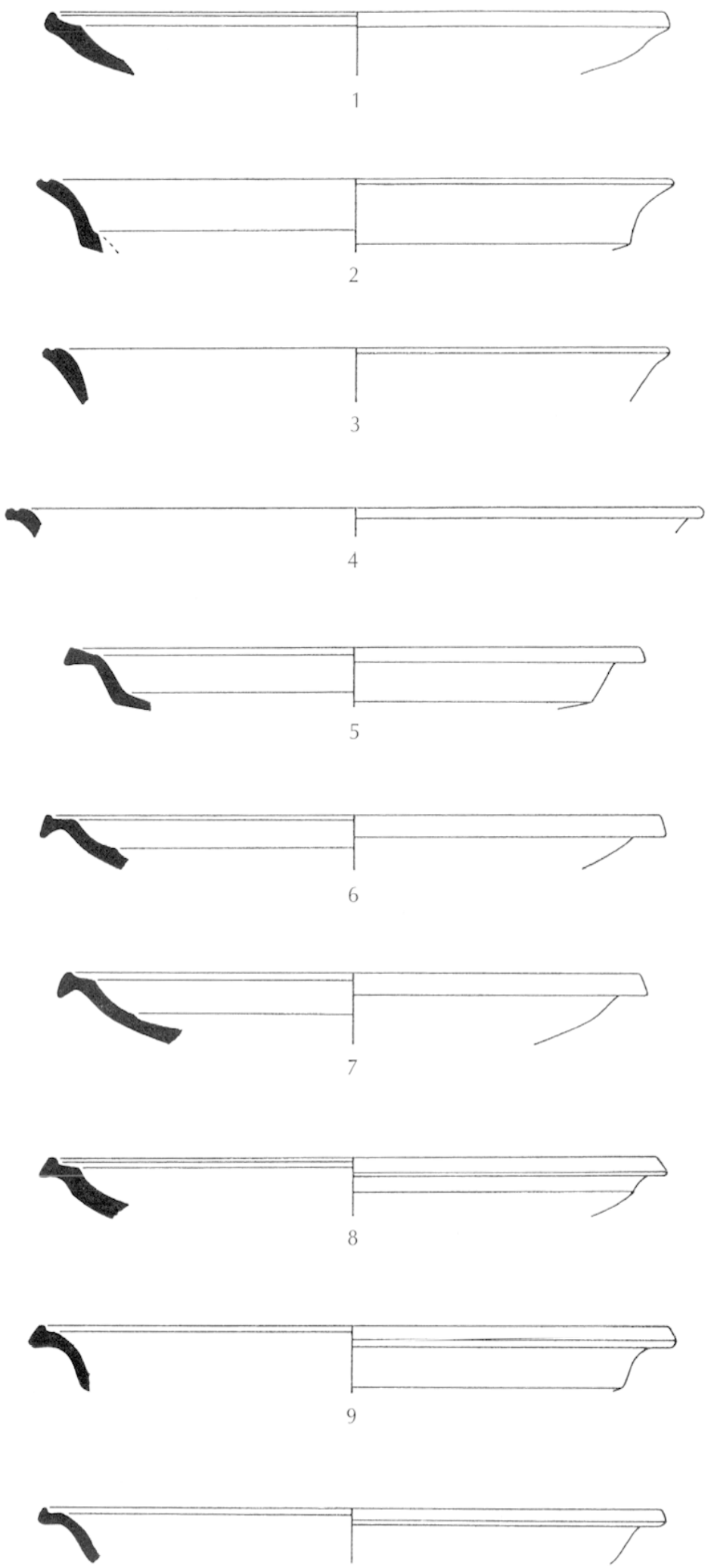

10

Pl. 2. Imitations de sigillée: formes apparentées au service I (échelle: $1 / 2$ ). 

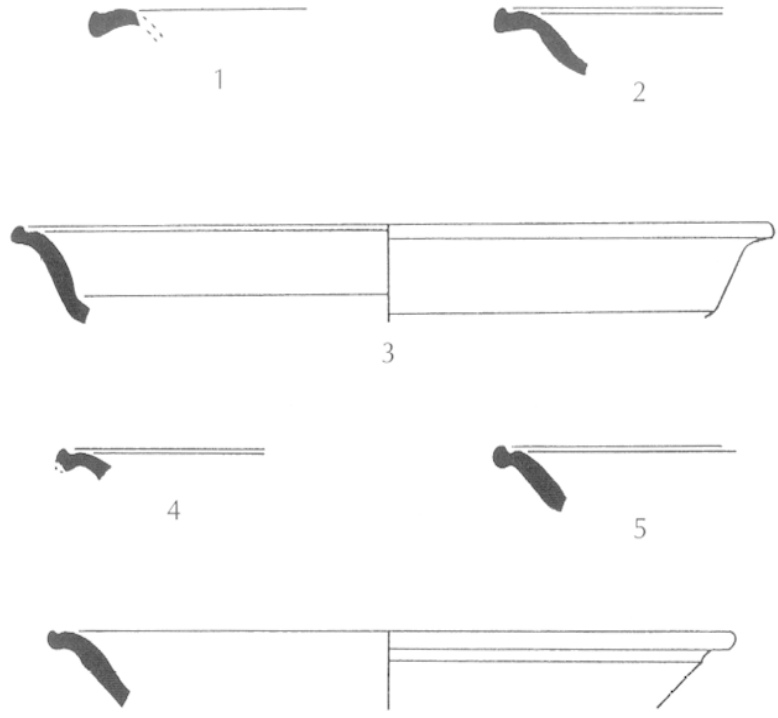

6
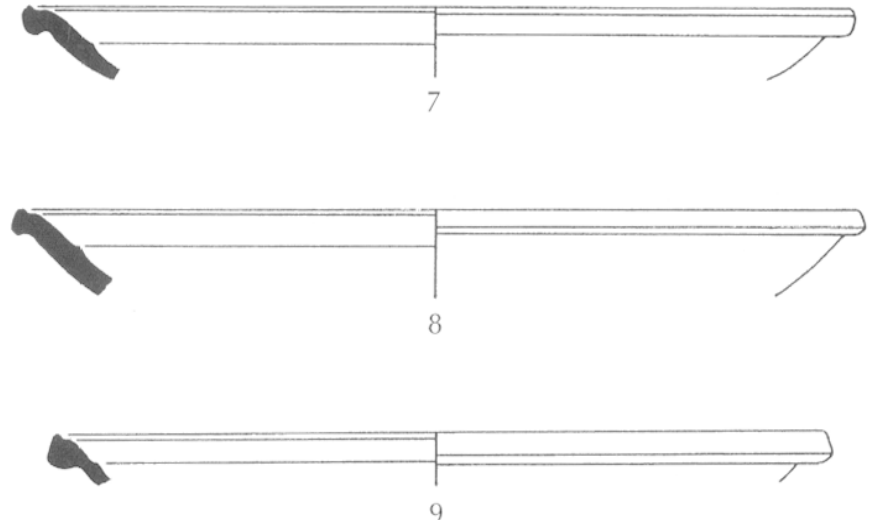

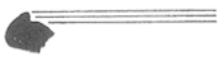

10

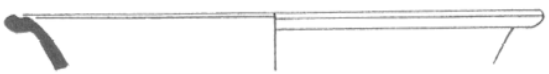

12

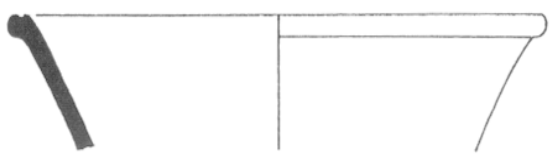

14
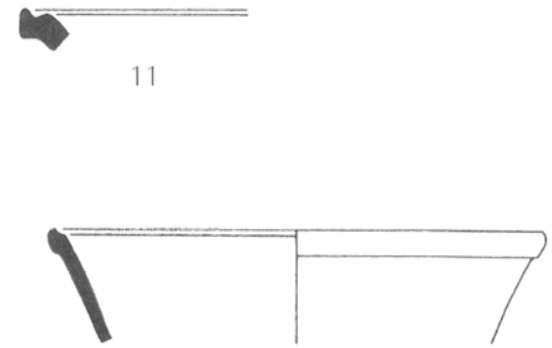

13

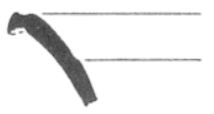

15

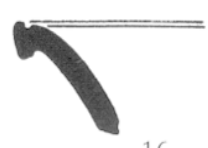

16

Pl. 3. Imilations de sigillée : formes apparentées au service I (échelle: 1/2). 

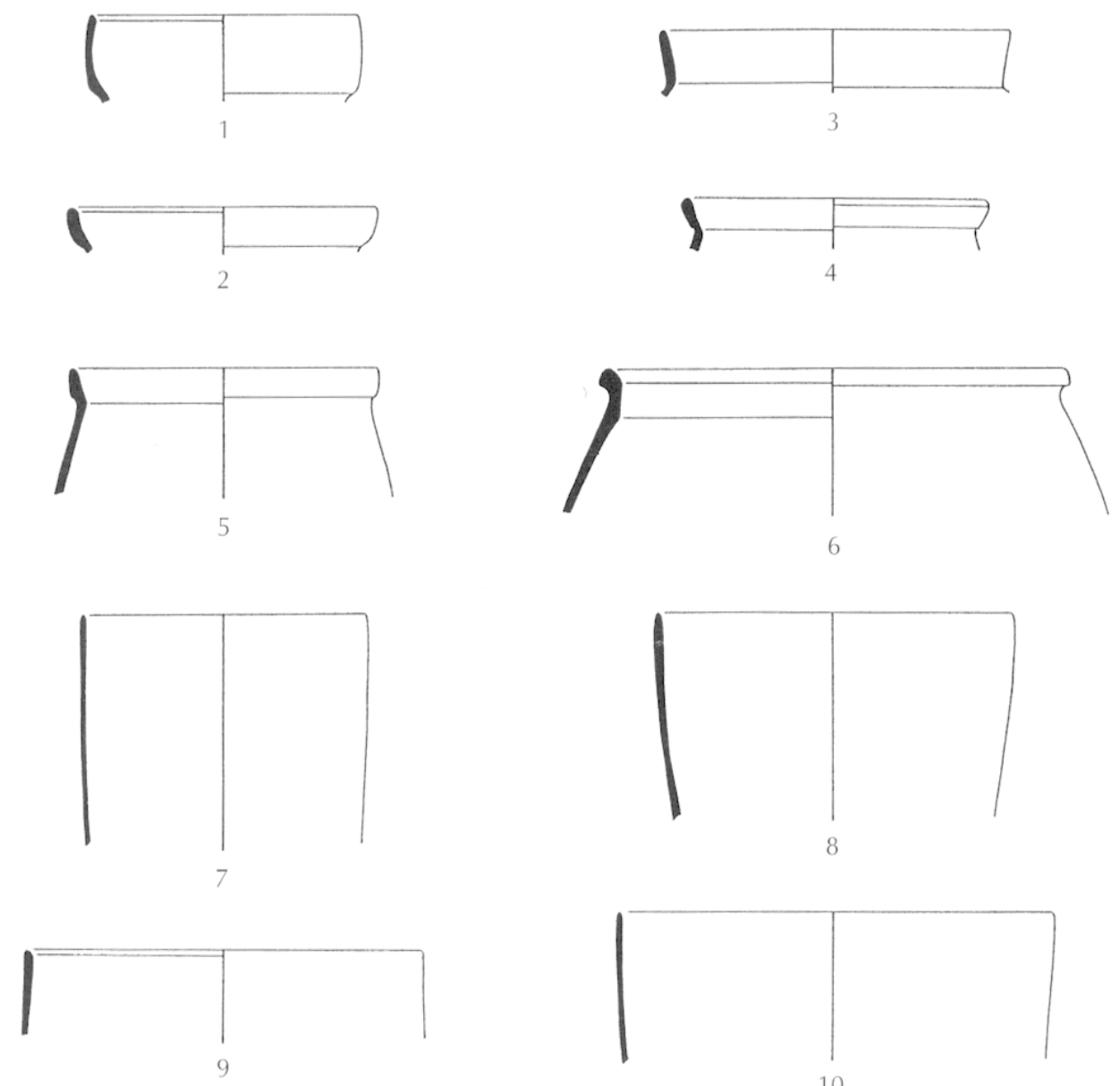

10

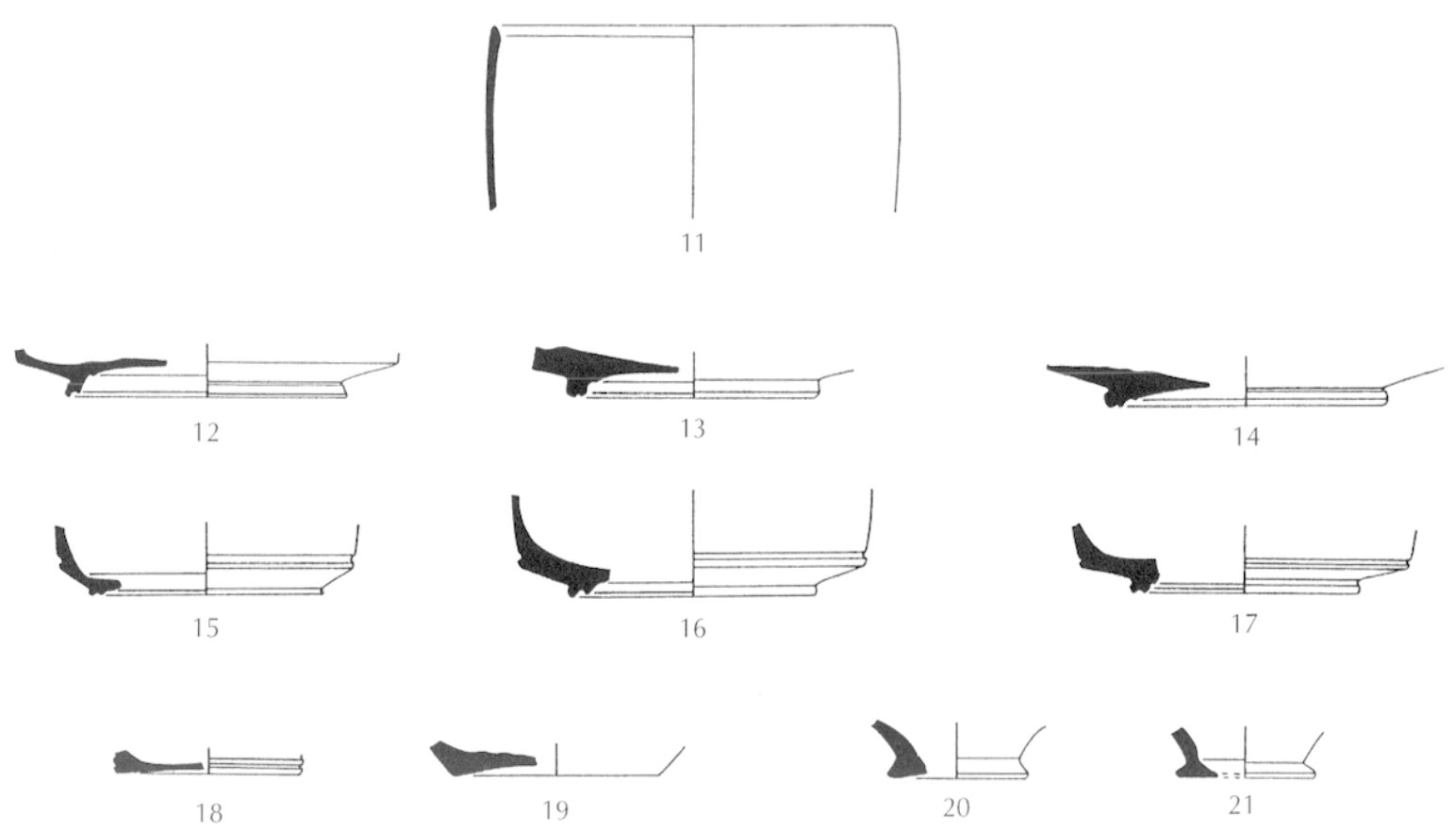

P1. 4. Vases à parois fines lisses (échelle: $1 / 2$ ). 

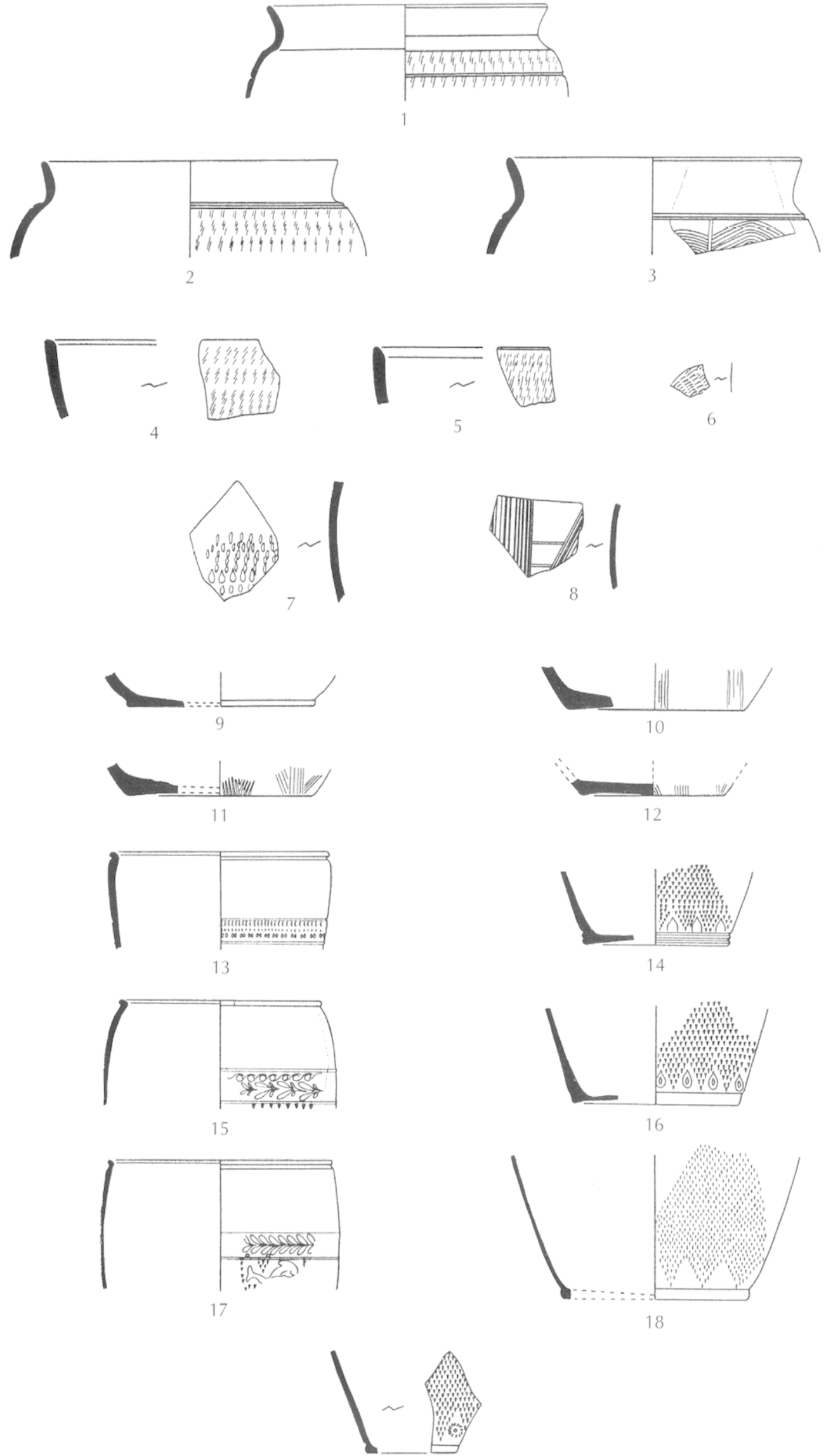

19

P1. 5. Vases à décor guilloché ou peigné (1-12) et gobelets d'Aco (13-19) (échelle : 1/2). 

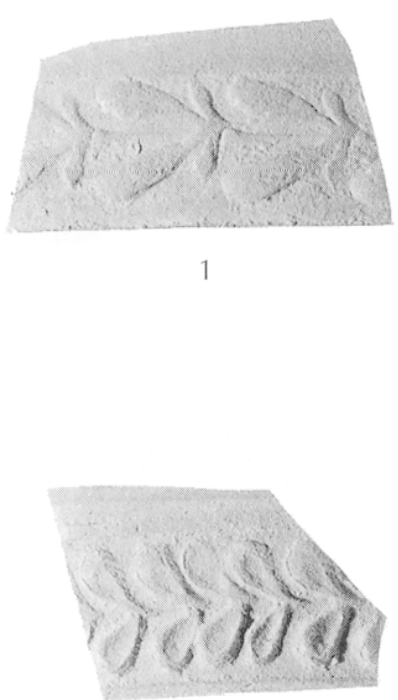

4

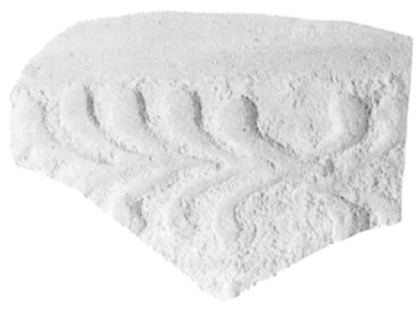

7

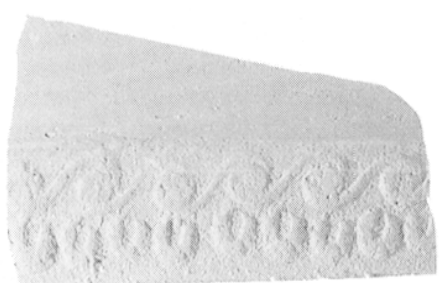

10

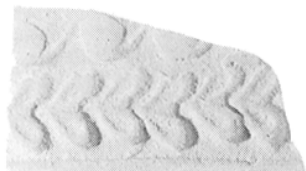

13
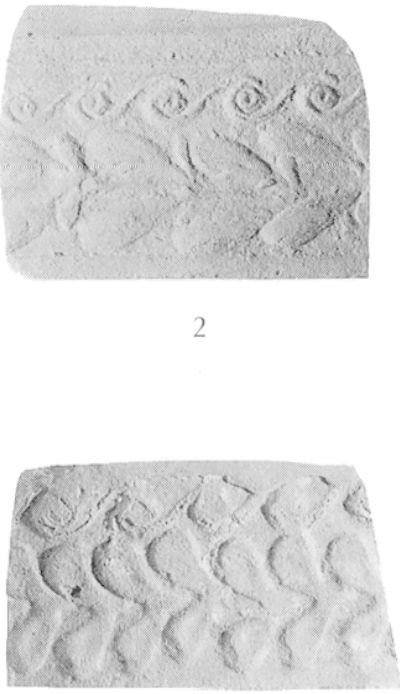

5
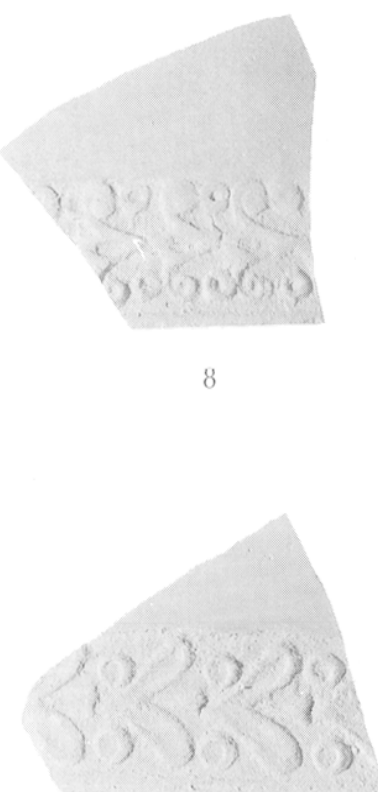

11

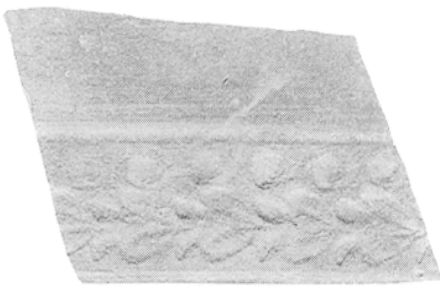

14
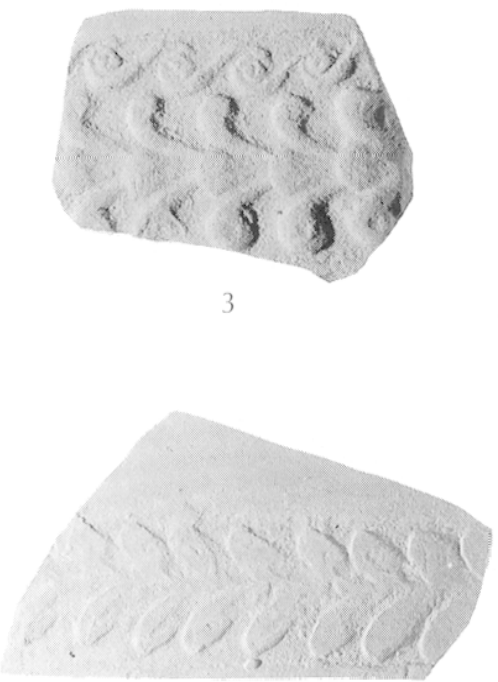

6

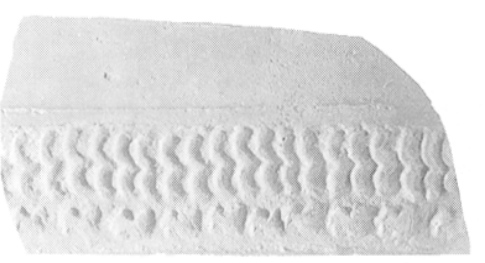

9

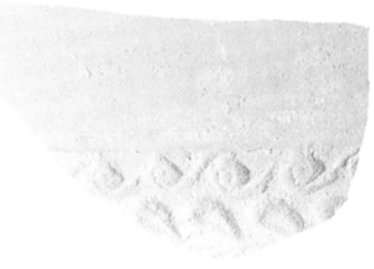

12

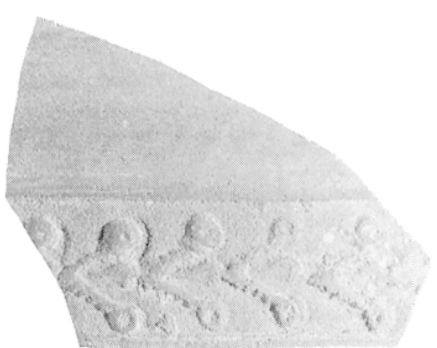

15

Pl. 6. Frises supérieures de gobelets d'Aco (échelle : 2/1). 

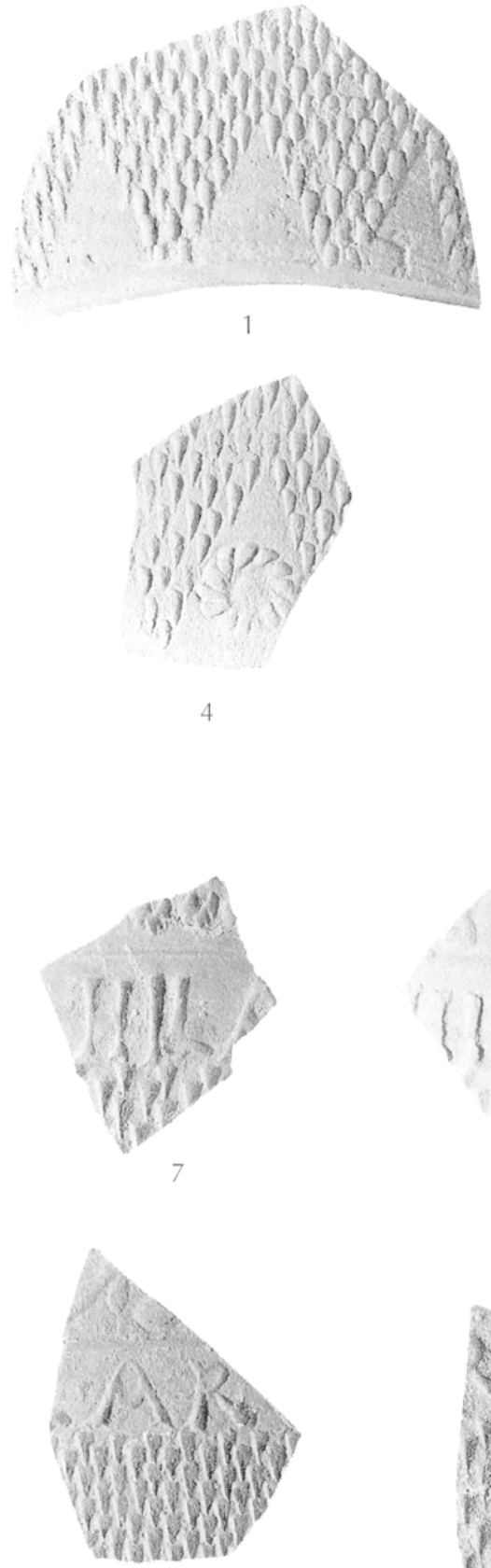

11
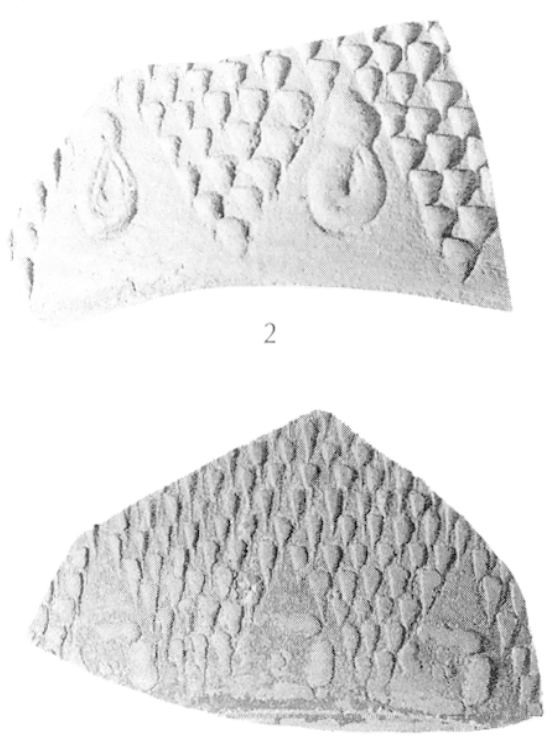

5
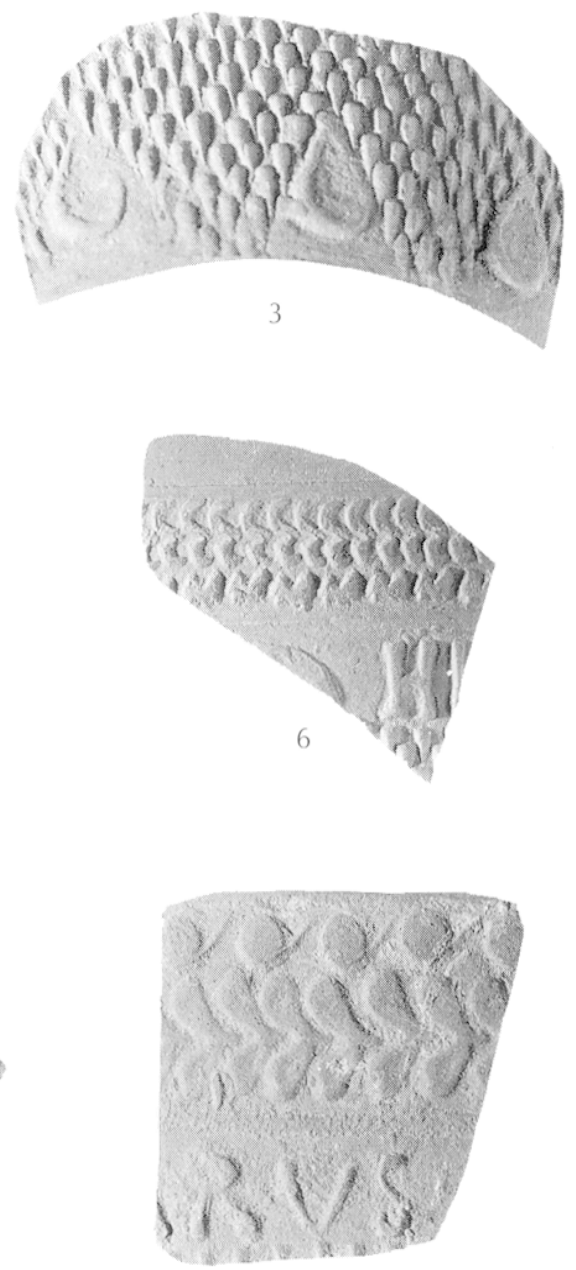

10

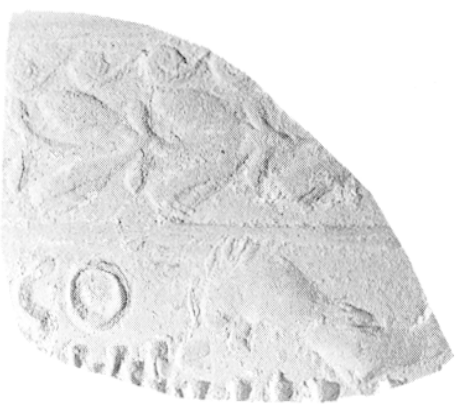

14

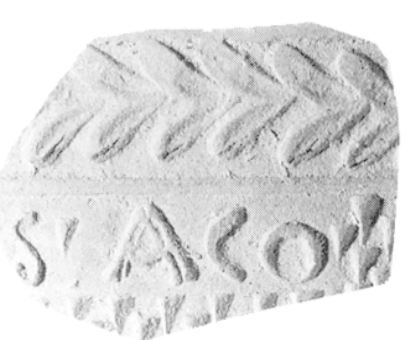

15
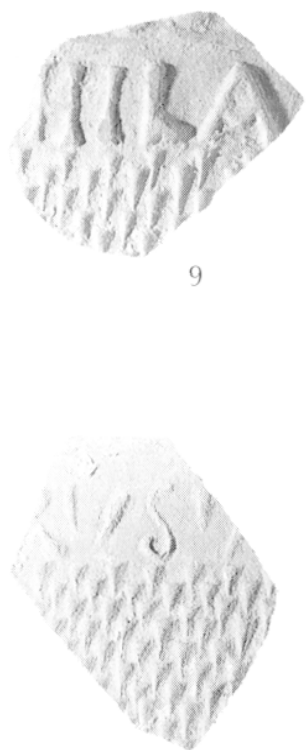

13

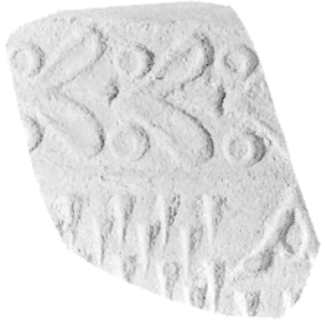

16

P1. 7. Motifs décorés sur fonds de gobelets d'Aco (1-5) et signatures sur frises (6-16) (échelle: 2/1). 


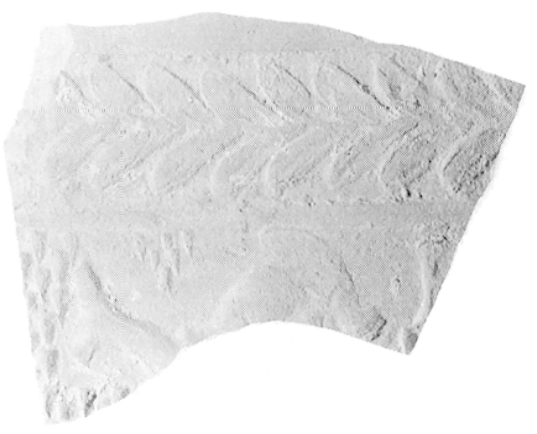

gauche 1

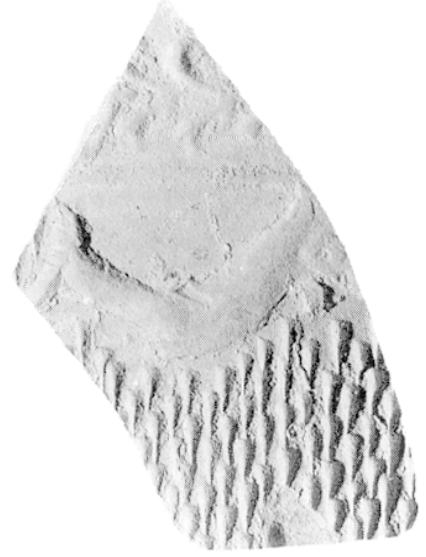

gauche 2

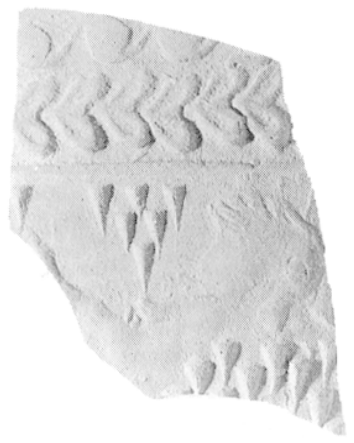

gauche 3

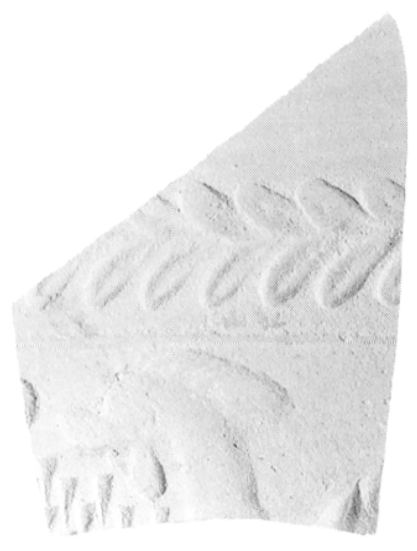

droite 2

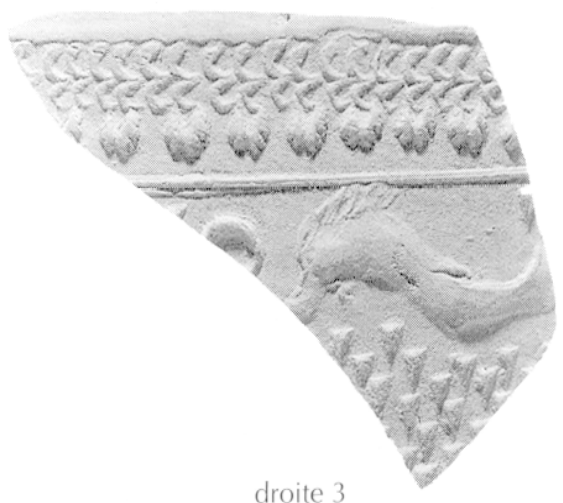

Pl. 8. Dauphins sur gobelets d'Aco (échelle : 2/1). 